\title{
Level Densities of Heavy Nuclei and Fission Dynamics
}

\author{
A. Rahmatinejad ${ }^{1}$, T. Shneidman ${ }^{1}$, G. Adamian ${ }^{1}$, \\ N.V. Antonenko ${ }^{1}$, P. Jachimowicz ${ }^{2}$, M. Kowal ${ }^{3}$ \\ ${ }^{1}$ Joint Institute for Nuclear Research, Dubna, 141980, Russia \\ ${ }^{2}$ Institute of Physics, University of Zielona Góra, \\ Szafrana 4a, 65516 Zielona Góra, Poland \\ ${ }^{3}$ National Centre for Nuclear Research, Pasteura 7, 02-093 Warsaw, Poland \\ Received 14 October 2021 \\ doi: https://doi.org/10.55318/bgjp.2021.48.5-6.485
}

\begin{abstract}
The nuclear level densities and level-density parameters in fissioning nuclei at their saddle points of fission barriers, as well as those for neutron, proton, and $\alpha$-particle emission residues at the ground states are calculated for isotopic chains of superheavy nuclei with $Z=114-$ 120. The calculations are performed with superfluid formalism using the single-particle energies obtained with the Woods-Saxon potential. The influence of shell effects on energy dependence of the ratios of leveldensity parameters corresponding to the residues of considered decay modes to those of neutron emission is analyzed. The effect of collective enhancement of level densities on the level-density parameter ratios is studied. In the case of alpha decay, we identified the collective enhancement caused by cluster degrees of freedom to play an important role.
\end{abstract}

KEY WORDS: fission barrier, level-density parameter, survival probability, superheavy nuclei, collective enhancements.

\section{Introduction}

The nuclear level density (NLD) is of special importance in the cross section calculations and determination of the survival probabilities. Going from low to higher energies the nuclear system reverts from a paired system to a system of noninteracting fermions which is successfully described by the well-known Fermi gas model (FGM) [1]. The level-density parameter $a$ which establishes the connection between excitation energy and nuclear temperature in FGM is often assumed to depend linearly on the mass number $A$ [2]. However, the level-density parameter is energy dependent and gradually reaches an asymptotic value at the energies higher than neutron separation energy, because of 


\section{A. Rahmatinejad, et al.}

internal structure of nucleus. Thus, for more realistic studies one can keep using the simple analytical expression of FGM with an energy dependent level density parameter by treating the pairing and shell effects in the frame of superfluid formalism [3,4]. In Ref. [5], the energy $U$, and shell-correction $\delta E$ dependencies of level-density parameter were studied for superheavy nuclei (SHN) at the ground state (GS) and the saddle point (SP) and compared with the phenomenological model. The level-density parameter ratios are important for the estimation of probabilities of de-excitation via light particles emission in competition with fission and thus for the determination of the survival probabilities. In the present work, the energy dependence of ratios of the level-density parameters corresponding to the residues of various decay channels relative to neutron emission is analyzed.

Knowing the collective states, one can directly take into account the collective enhancement of NLDs. Alternative way is to use the phenomenological expression introduced by Ignatyuk [6] in the form of a product of vibrational and rotational coefficients. The collective enhancements of the level densities were calculated and compared with those of the phenomenological model for well deformed isotopes of Dy and almost spherical isotopes of Mo in Ref. [7]. It was shown that the phenomenological expression of collective enhancement works well for the case of well deformed nuclei [7]. The collective enhancement of level density is connected with the shape of nucleus. Because the nucleus at SP is strongly deformed, additional collective states related to rotational excitations are expected with respect to the GS. Also, the level density for $\alpha$-emission channel can be enhanced because of additional collective states related to the cluster degrees of freedom $[8,9]$. In this work the effect of collective enhancement of level densities on the level-density parameter ratios is studied.

\section{Calculation Formalism}

Based on the superfluid formalism [3,4] and with an assumption of thermal equilibrium between neutron and proton subsystems, the excitation energies $U=U_{Z}+U_{N}$, entropies $S=S_{Z}+S_{N}$ and intrinsic level densities $\rho$ are calculated as

$$
\begin{aligned}
& E_{Z(N)}(T)=\sum_{k} \varepsilon_{k}\left(1-\frac{\varepsilon_{k}^{Z(N)}-\lambda_{Z(N)}}{E_{k}^{Z(N)}} \tanh \frac{\beta E_{k}^{Z(N)}}{2}\right)-\frac{\Delta_{Z(N)}^{2}}{G_{Z(N)}}, \\
& U_{Z(N)}(T)=E_{Z(N)}(T)-E_{Z(N)}(0), \\
& S_{Z(N)}(T)=\sum_{k}\left\{\ln \left[1+\exp \left(-\beta E_{k}^{Z(N)}\right)\right]+\frac{\beta E_{k}^{Z(N)}}{1+\exp \left(\beta E_{k}^{Z(N)}\right)}\right\}, \\
& \rho_{i}=\frac{\exp (S)}{(2 \pi)^{\frac{3}{2}} \sqrt{D}}
\end{aligned}
$$


where $D$ is the determinant of the matrix comprised of the second derivatives of the entropy with respect to $\beta$ and $\mu_{Z(N)}=\beta \lambda_{Z(N)}$,

$$
D=\left|\begin{array}{ccc}
\frac{\partial^{2} S}{\partial \beta^{2}} & \frac{\partial^{2} S}{\partial \beta \partial \mu_{Z}} & \frac{\partial^{2} S}{\partial \beta \partial \mu_{N}} \\
\frac{\partial^{2} S}{\partial \beta \partial \mu_{Z}} & \frac{\partial^{2} S}{\partial \mu_{Z}^{2}} & 0 \\
\frac{\partial^{2} S}{\partial \beta \partial \mu_{N}} & 0 & \frac{\partial^{2} S}{\partial \mu_{N}^{2}}
\end{array}\right| .
$$

The quasiparticle energies $E_{k}^{Z(N)}=\sqrt{\left(\varepsilon_{k}^{Z(N)}-\lambda_{Z(N)}\right)^{2}+\Delta_{Z(N)}^{2}}$ are obtained using the single-particle energies $\left(\varepsilon_{k}^{Z(N)}\right)$ calculated in the microscopicmacroscopic model based on the deformed single-particle Woods-Saxon potential and Yukawa-plus exponential macroscopic energy $[10,11]$. The constants of the pairing interaction for neutrons $\left(G_{N}\right)$ and protons $\left(G_{Z}\right)$ are adjusted to obtain the GS pairing gaps $\left(\Delta_{N}\right.$ and $\left.\Delta_{Z}\right)$ at zero temperature with BardeenCooper-Schrieffer (BCS) equations

$$
\begin{gathered}
Z(N)=\sum_{k}\left(1-\frac{\varepsilon_{k}^{Z(N)}-\lambda_{Z(N)}}{E_{k}^{Z(N)}} \tanh \frac{\beta E_{k}^{Z(N)}}{2}\right), \\
\frac{2}{G_{Z(N)}}=\sum_{k} \frac{1}{E_{k}^{Z(N)}} \tanh \frac{\beta E_{k}^{Z(N)}}{2}
\end{gathered}
$$

where $Z(N)$ is the numbers of protons(neutrons) in the nucleus. In our calculations, in the cases of $\Delta_{Z(N)}<0.25 \mathrm{MeV}$ the pairing gap is taken as $0.25 \mathrm{MeV}$. Using the values of pairing constants obtained, the pairing gaps and chemical potentials $\lambda_{Z(N)}$ are determined by solving Eqs. (6), and (7) at given temperatures $T=1 / \beta$. The calculations are performed for the isotopic chains of nuclei with $Z=112-120$. In order to obtain the level densities of nuclei in fission channel, the calculations were repeated for the same nuclei using the single-particle energies obtained with the Woods-Saxon potential at the SP. In the BCS calculations at SP, the pairing constants were taken as in the GS.

Fitting the calculated intrinsic level density in the interval between 10 and $100 \mathrm{MeV}$ with the back-shifted Fermi gas expression [1]

$$
\rho_{F G}(U)=\frac{\sqrt{\pi}}{12 a^{1 / 4}(U-\Delta)^{5 / 4}} \exp (2 \sqrt{a(U-\Delta)}),
$$

the energy dependent level-density parameter $a(U)$ is obtained. The energy back-shifts in Eq. (8) are taken as $\Delta=12 / \sqrt{A}, 0,-12 / \sqrt{A}$ for even-even, odd- $A$ and odd-odd isotopes, respectively. 


\section{A. Rahmatinejad, et al.}

In the level density parameter ratios defined as

$$
\begin{aligned}
& \frac{a_{f}}{a_{n}}=\frac{a_{S P}\left(A, U-B_{f}\right)}{a_{G S}\left(A-1, U-B_{n}\right)}, \\
& \frac{a_{p}}{a_{n}}=\frac{a_{G S}\left(A-1, U-B_{p}\right)}{a_{G S}\left(A-1, U-B_{n}\right)}
\end{aligned}
$$

and

$$
\frac{a_{\alpha}}{a_{n}}=\frac{a_{G S}\left(A-4, U-B_{\alpha}\right)}{a_{G S}\left(A-1, U-B_{n}\right)},
$$

the energy costs due to fission barrier $B_{f}$, neutron separation energy $B_{n}$, as well as proton $B_{p}=V_{c}^{p}-Q_{p}$, and $\alpha$-particle $B_{\alpha}=V_{c}^{\alpha}-Q_{\alpha}$ energy thresholds are taken into consideration. Here,

$$
V_{c}^{p, \alpha}=\frac{1.44\left(Z-Z_{p, \alpha}\right) Z_{p, \alpha}}{C_{p, \alpha}\left[\left(A-A_{p, \alpha}\right)^{1 / 3}+A_{p, \alpha}^{1 / 3}\right]}
$$

are the Coulomb barriers for charged particles, where $Q_{p, \alpha}$-values are taken from microscopic-macroscopic model [12]. In Eq. (12), $A_{p}=Z_{p}=1, A_{\alpha}=$ $2 Z_{\alpha}=4$, and the constants $C_{p, \alpha}$ are taken as $C_{p}=1.7 \mathrm{fm}$ and $C_{\alpha}=1.57 \mathrm{fm}$ [13].

A way to take into account the collective enhancement of nuclear level density is to use an adiabatic approximation [14]:

$$
\rho(U)=\rho_{i}(U) K_{\text {coll }} .
$$

Knowing the collective excitation energies $U_{c}$ and their degeneracies $\tau_{c}$ the collective enhancement $K_{\text {coll }}$ can be obtained as [7]

$$
K_{\text {coll }}(\beta)=\sum_{c} \exp \left(-\beta U_{c}\right) \tau_{c} .
$$

Alternative way is to use the phenomenological expression in the form of [14]

$$
K_{\text {coll }}=K_{\text {rot }} K_{\text {vib }} \text {. }
$$

The enhancement due to rotational degrees of freedom is expressed as $[14,15]$

$$
K_{\text {rot }}= \begin{cases}1, & \text { for spherical nuclei } \\ \Im_{\perp} T, & \text { for deformed nuclei, }\end{cases}
$$

where, $\Im_{\perp}=\Im_{r . b} f\left(\beta_{2}, \beta_{4}\right)$ is the moment of inertia with respect to the axis perpendicular to the symmetry axis. Here, $f\left(\beta_{2}, \beta_{4}\right)=1+\sqrt{5 / 16 \pi} \beta_{2}+$ $(45 / 28 \pi) \beta_{2}^{2}+\left(15 / 7 \pi \sqrt{5} \beta_{2} \beta_{4}\right)$, where $\beta_{2}$ and $\beta_{4}$ are quadrupole and hexadecapole deformation parameters of the nucleus. The liquid-drop model estimation for the vibrational enhancement $[14,15]$ is

$$
K_{\text {vib }}=\exp \left(0.0555 A^{2 / 3} T^{4 / 3}\right) .
$$




\section{Results}

The excitation energy dependence of $a_{f} / a_{n}$ is displayed in Figure 1(a) for the 295-302 120 isotopic chain. The energy dependence of $a_{f} / a_{n}$ was shown and discussed in Ref. [5]. Generally, the $a_{f} / a_{n}$ ratio grows fast to a maximum and then slowly falls to an asymptotic value less than 1.1. Depending on the difference between the shell-corrections at the SP and at the GS together with their damping rates, the values of $a_{f} / a_{n}$ for various isotopes reach maxima at different energies but lower than $25 \mathrm{MeV}$. For some cases such as $296,298,302120$, the shell effect at saddle point fades out so fast that at energies where the $a_{f} / a_{n}$ ratios
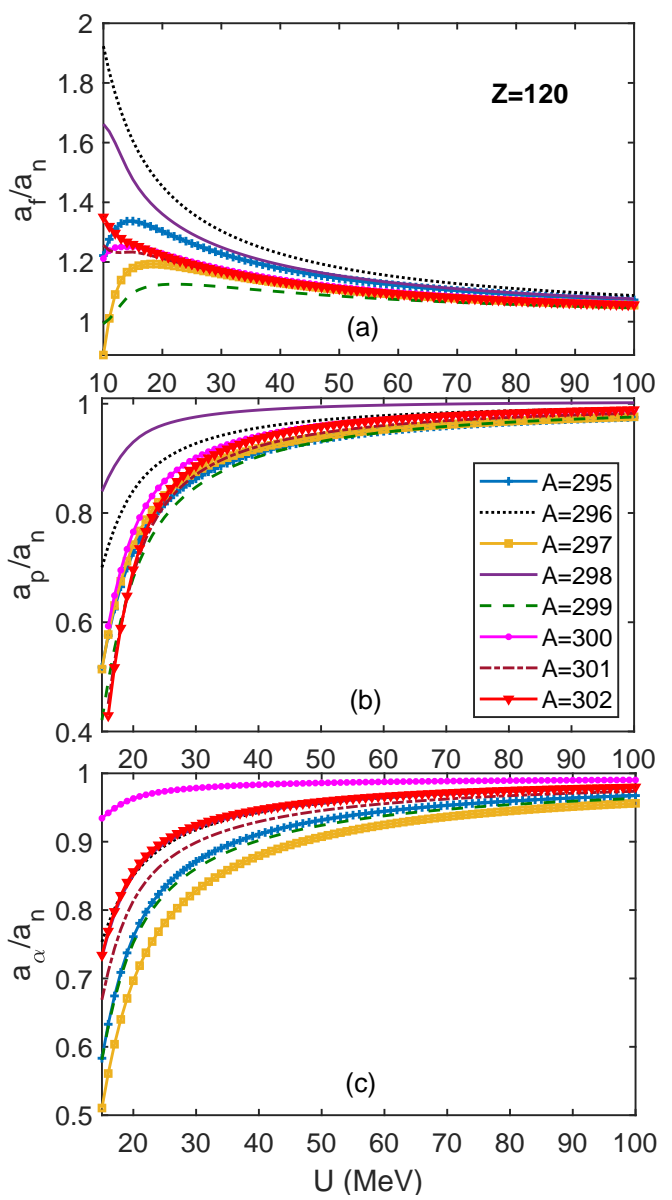

Figure 1. (Color online) Excitation energy dependence of $a_{f} / a_{n}$ (panel a), $a_{p} / a_{n}$ (panel b), and $a_{\alpha} / a_{n}$ (panel c) calculated with Eqs. (9-11) for ${ }^{295-302} 120$ isotopic chain. The mass number $A$ of the given isotope is indicated by the selected color and style. 
A. Rahmatinejad, et al.

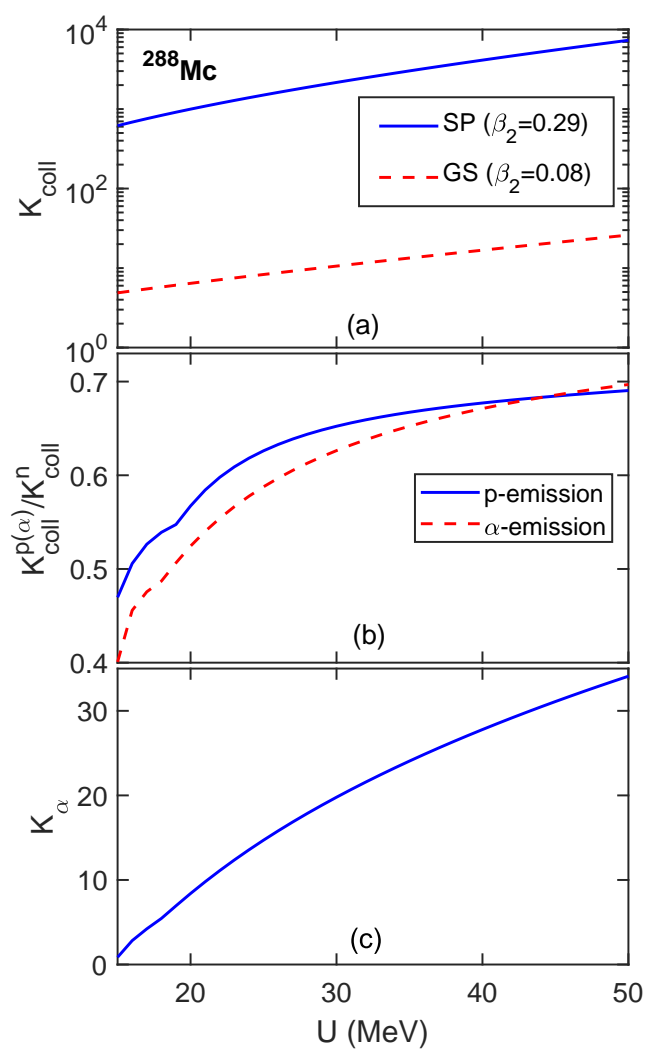

Figure 2. (Color online) (a): Excitation energy dependence of $K_{\text {coll }}$ calculated with Eq. (15) for ${ }^{288} \mathrm{Mc}$ at the saddle point (blue solid line) and the ground state (red dashed line). (b): The ratio of collective enhancement in level densities of proton ( $\alpha$-particle) emission residue to that of the neutron emission calculated with Eq. (15) at $\left(U-B_{\tau}\right)$. Here, $U$ is the excitation energy of mother nucleus ${ }^{288} \mathrm{Mc}$ and $B_{\tau}(\tau=p, \alpha, n)$ are the particle emission energy thresholds. (c): Collective enhancement caused by cluster degrees of freedom $K_{\alpha}$ calculated for the $\alpha$-decay residue versus the excitation energy of mother nucleus ${ }^{288} \mathrm{Mc}$.

are calculated, they appear already at their maximum and decrease monotonically with the excitation energy. In Figure 1(b) and (c), the energy dependencies of $a_{p} / a_{n}$ and $a_{\alpha} / a_{n}$ are shown for the same isotopes. Since the ground states have close rates of shell-correction damping, the relative difference between the level-density parameters of particle emission residues uniformly decreases with excitation energy. Therefore, the $a_{p} / a_{n}$ and $a_{\alpha} / a_{n}$ curves do not demonstrate maxima at low energies. For the most of considered isotopes the $a_{p(\alpha)} / a_{n}$ ratios approach an asymptotic value in the range of $0.95-1$. In Figure. 1, only the ratios of internal level densities are shown. 
The phenomenological expression of collective enhancement (15) works well for the case of well-deformed nuclei [7]. Hence, one can reliably apply it for the calculation of collective effects due to rotation and vibrations at SP where a nucleus is strongly deformed. Most of the isotopes considered in our calculations are nearly spherical at the GS with quadrupole deformation parameters less than 0.2 . As an example, the collective enhancements of level densities in ${ }^{288} \mathrm{Mc}$ at the GS and SP are displayed in Figure 2(a). For the GS with $\beta_{2}=0.08$, the rotational coefficient is taken as unity. However, this is applicable if the nucleus is assumed to be ideally spherical. As discussed in Ref. [7], despite the nearly spherical shape of a nucleus, the rotational enhancement is not totaly negligible. Therefore, since the rotational enhancement in neutron emission residue is expected to be less than that of the SP, to estimate relative importance of the channels it should be taken into consideration.

For the particle emission channels, the collective enhancements due to rotational and vibrational degrees of freedom are similar in daughter nuclei because of their similar deformations. The ratio of collective enhancement in level densities of proton and $\alpha$-particle emission to that of the neutron emission is displayed in Figure 2(b) for ${ }^{288} \mathrm{Mc}$. The separation energies and Coulomb barrier are taken into account for the calculation of $K_{\text {coll }}$. As seen from the figure, the effect of collective enhancement can be neglected.

If the effect of collective enhancement of level density is known in $a_{f} / a_{n}$, we suggest to take into account the additional collective effects on $\alpha$-emission channel $\left(K_{\alpha}\right)$ as well. This can be done in the same way as Eq. (14) but with the collective excited states due to cluster degrees of freedom $[8,9]$

$$
U_{c}=\hbar \omega_{m a} n_{m a}+\hbar \omega_{b}\left(2 n_{b}+|K|\right)
$$

and degeneracies $\tau_{c}=2|K|+1$. Here, $\hbar \omega_{m a}$ is the frequency of vibrations in mass asymmetry while $\hbar \omega_{b}$ describes the relative vibrations of $\alpha$ particle and daughter nucleus, and $n_{b}, n_{m a}$, and $K$ are the corresponding quantum numbers. In Figure 2(c), the collective enhancement caused by cluster degrees of freedom in the $\alpha$-decay channel is shown versus the excitation energy of the mother nucleus ${ }^{288} \mathrm{Mc}$. Taking $\hbar \omega_{m a}=0.5 \mathrm{MeV}$, and $\hbar \omega_{b}=0.3 \mathrm{MeV}$ we find $K_{\alpha}(\beta)$ values varying up to 35 in the energy range of $15-50 \mathrm{MeV}$. One can take effectively into account this enhancement with $K_{\alpha} \approx 20$. This allows the $a_{\alpha} / a_{n}$ ratios to reach asymptotic values larger than unity. Excitation energy dependencies of $a_{\alpha} / a_{n}$ ratios calculated for ${ }^{291-298} \mathrm{Ts}$, and ${ }^{295-302} 119$ nuclei with and without account of $K_{\alpha}(\beta)$ are shown in Figure 3 panels (a-e). The account of $K_{\alpha}$ leads not only to an increase in the absolute value of $a_{\alpha} / a_{n}$, but also to a functional change in the dependence of $a_{\alpha} / a_{n}$ on $U$. For example, the maximum of the ratio $a_{\alpha} / a_{n}$ occurs in the region of energies of 20-30 MeV. 
A. Rahmatinejad, et al.

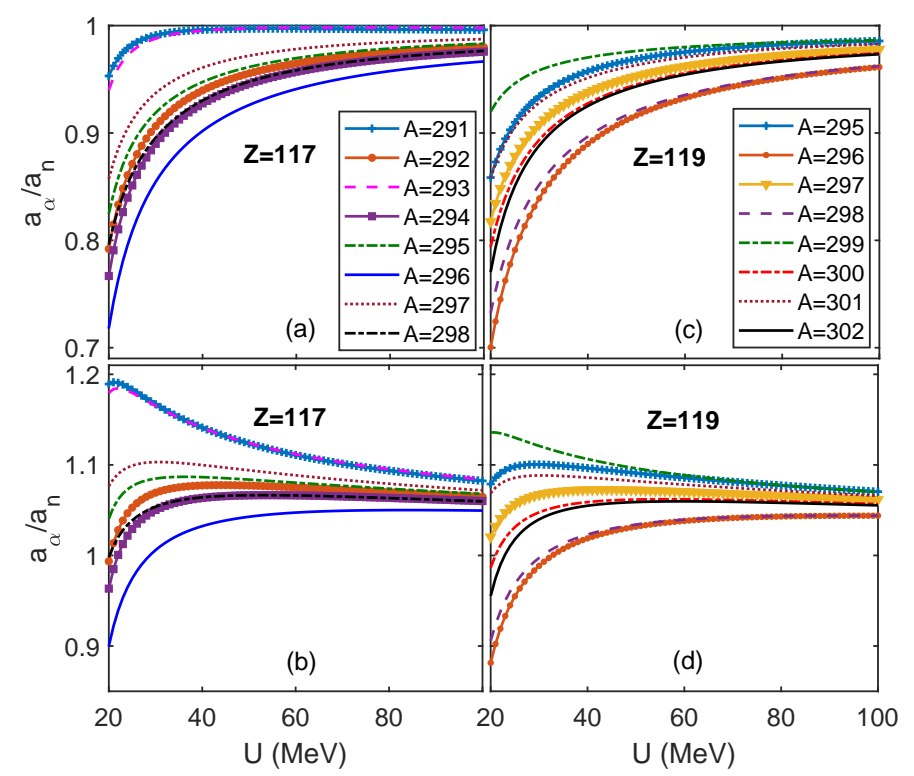

Figure 3. (Color online) The ratios $a_{\alpha} / a_{n}$ calculated for ${ }^{291-298} \mathrm{Ts}$, and ${ }^{295-302} 119$ nuclei with (panels b and d) and without (panels a and c) account of collective enhancement due to cluster degrees of freedom $K_{\alpha}$ in the level densities of $\alpha$ emission residues, versus the excitation energies of mother nuclei. The mass number $A$ of the given isotope is indicated by the selected color and style.

\section{Summary}

The intrinsic level densities of SHN with $Z=112-120$ at the ground state and at the saddle point are calculated within the thermodynamic superfluid formalism. The single-particle energies, shell corrections and nuclear masses used in these calculations were obtained within the multidimensional microscopicmacroscopic model [12]. The energy dependent level-density parameters of the considered nuclei were obtained by fitting the calculated intrinsic level densities with the Fermi-gas expression. Then, the energy dependent ratios of leveldensity parameters corresponding to the nuclei at the fission saddle point $a_{f}$, and proton $a_{p}$ and $\alpha$-particle $a_{\alpha}$ emission residues at their GS to the ones obtained for the daughter nuclei after neutron emission $a_{n}$ were calculated. Generally, the level-density parameter ratios reach an asymptotic value less than 1.1 for $a_{f} / a_{n}$, and less than unity for $a_{p, \alpha} / a_{n}$. Our analysis shows important effects of decay thresholds and shell correction on the energy dependence of level-density parameter ratios. Because of large difference in the shell corrections at the SP and at the GS as well as different rates of their damping with excitation energy, the ratios $a_{f} / a_{n}$ demonstrate a maximum at an energy lower than $25 \mathrm{MeV}$. Because 
Level Densities of Heavy Nuclei and Fission Dynamics

of close values of shell corrections and their damping rates at the ground states, the ratios $a_{p} / a_{n}$ and $a_{\alpha} / a_{n}$ monotonically increase with excitation energy. The account of collective effects due to cluster degrees of freedom in the level densities of $\alpha$-emission residue enhances the ratio $a_{\alpha} / a_{n}$ to the values larger than unity. So, the values of $a_{\alpha} / a_{n}$ and $a_{f} / a_{n}$ larger than those obtained microscopically effectively account the collective enhancement of level density. The information and analysis performed in this article can be useful for the calculation of energy dependent ratios of the widths for the considered decay modes and further applied to evaluate corresponding cross sections and probability of survival against fission and charged particle emissions.

\section{Acknowledgements}

This work was supported by by the RFBR grant 20-02-00176 and by the PolishJINR cooperation program.

\section{References}

[1] H. Bethe (1937) Nuclear physics B. nuclear dynamics, theoretical. Rev. Mod. Phys. 969.

[2] Yu.V. Sokolov (1937) "Level Densities of Atomic Nuclei" (Energoizdat, Moscow).

[3] P. Decowski, W. Grochulski, A. Marcinkowski, K. Siwek, Z. Wilhelmi (1968) Isomeric cross-section ratios and total cross sections for the ${ }^{59} \mathrm{Co}(\mathrm{n}, 2 \mathrm{n})^{58 g, m} \mathrm{Co}$, ${ }^{58} \mathrm{Ni}(\mathrm{n}, \mathrm{p}){ }^{58 g, m} \mathrm{Co}$ AND ${ }^{59} \mathrm{Co}(\gamma, \mathrm{n}){ }^{58 g, m}$ Co reactions. Nucl. Phys. A 110129. DOI: https://doi.org/10.1016/0375-9474(68)90075-4.

[4] G.D. Adeev and P.A. Cherdantsev (1975) Application of the Strutinskii Method to the Investigation of Shell Effects in the Density of Excited Nuclear States. Yad. Fiz. 21491.

[5] A. Rahmatinejad, A.N. Bezbakh, T.M. Shneidman, G. Adamian, N.V. Antonenko, P. Jachimowicz, and M. Kowal (2021) Level-density parameters in superheavy nuclei Phys. Rev. C 103 034309 DOI: https://doi.org/10.1103/PhysRevC.103.034309.

[6] A.B. Ignatyuk, G.N. Smirenkin, A.S. Tishin (1975) Phenomenological description of the energy dependence of the level density parameter Yad. Fiz. 21485.

[7] A. Rahmatinejad, T.M. Shneidman, N.V. Antonenko, A.N. Bezbakh, G.G. Adamian, and L.A. Malov (2020) Collective enhancements in the level densities of Dy and Mo isotopes Phys. Rev. C 101054315 DOI: https://doi.org/10.1103/ PhysRevC.101.054315.

[8] T.M. Shneidman, G.G. Adamian, N.V. Antonenko, R.V. Jolos, and Shan-Gui Zhou (2015) Cluster approach to the structure of ${ }^{240} \mathrm{Pu}$ Phys. Rev. C 92034302 DOI: https://doi.org/10.1103/PhysRevC.92.034302.

[9] T.M. Shneidman, G.G. Adamian, N.V. Antonenko, R.V. Jolos, and W. Scheid (2003) Cluster interpretation of properties of alternating parity bands in heavy nuclei Phys. Rev. C 67014313 DOI: https://doi.org/10.1103/PhysRevC.67.014313.

[10] M. Kowal, P. Jachimowicz, and A. Sobiczewski (2010) Fission barriers for eveneven superheavy nuclei Phys. Rev. C 82014303 DOI: https://doi.org/10.1103/ PhysRevC.82.014303. 


\section{A. Rahmatinejad, et al.}

[11] P. Jachimowicz, M. Kowal, and J. Skalski (2017) Adiabatic fission barriers in superheavy nuclei Phys. Rev. C 95014303 DOI: https://doi.org/10.1103/ PhysRevC.95.014303.

[12] P. Jachimowicz, M. Kowal, J. Skalski (2021) Properties of heaviest nuclei with $98 \leq$ $\mathrm{Z} \leq 126$ and $134 \leq \mathrm{N} \leq 192$ Atomic Data and Nuclear Data Tables 138101393 DOI: https://doi.org/10.1016/j.adt.2020.101393.

[13] Juhee Hong, G.G. Adamian, and N.V. Antonenko (2016) Possibilities of production of transfermium nuclei in charged-particle evaporation channels Phys. Rev. C 94 044606 DOI: https://doi.org/10.1103/PhysRevC.94.044606.

[14] A.B. Ignatyuk (1983) "The statistical properties of the excited atomic nuclei" (Energoatomizdat, Moscow)

[15] A. S. Iljinov, M.V. Mebel, N. Bianchi, E. De Sancitis, C.Guaraldo, V. Lucherini, V. Muccifora, E. Polli, A.R. Re-olon, and P. Rossi (1992) Phenomenological statistical analysis of level densities, decay width and lifetime of excited nuclei Nucl. Phys. A 543517 DOI: https://doi.org/10.1016/0375-9474(92)90278-R. 